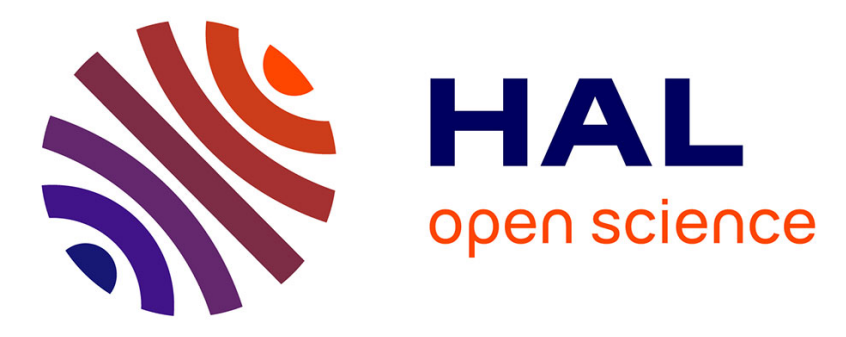

\title{
Analyse électromyographique de la production des plosives labiales: enjeux méthodologiques
}

Thibault Cattelain, Maëva Garnier, Christophe Savariaux, Silvain Gerber, Pascal Perrier

\section{- To cite this version:}

Thibault Cattelain, Maëva Garnier, Christophe Savariaux, Silvain Gerber, Pascal Perrier. Analyse électromyographique de la production des plosives labiales: enjeux méthodologiques. JEP 2018 - 32e Journées d'Etudes sur la Parole, Jun 2018, Aix-en-Provence, France. hal-01867567

\section{HAL Id: hal-01867567 https://hal.science/hal-01867567}

Submitted on 4 Sep 2018

HAL is a multi-disciplinary open access archive for the deposit and dissemination of scientific research documents, whether they are published or not. The documents may come from teaching and research institutions in France or abroad, or from public or private research centers.
L'archive ouverte pluridisciplinaire HAL, est destinée au dépôt et à la diffusion de documents scientifiques de niveau recherche, publiés ou non, émanant des établissements d'enseignement et de recherche français ou étrangers, des laboratoires publics ou privés. 


\title{
Analyse électromyographique de la production des plosives labiales : enjeux méthodologiques.
}

\author{
Thibault Cattelain, Maëva Garnier, Christophe Savariaux, Silvain Gerber, Pascal Perrier \\ Univ. Grenoble Alpes, CNRS, Grenoble INP*, GIPSA-lab, 38000 Grenoble, France \\ * Institute of Engineering Univ. Grenoble Alpes \\ thibault.cattelain@gipsa-lab.grenoble-inp.fr
}

\section{RESUME}

Notre objectif est de montrer l'intérêt d'une d'analyse temporelle détaillée des gestes de production des consonnes occlusives labiales, en distinguant différentes phases, y inclus durant l'occlusion où les lèvres sont immobiles. Quatre adultes ont été enregistrés pour cette étude. Il leur était demandé de produire des logatomes contenant les plosives /p/ et /b/, dans différents contextes vocaliques (/a/, /i/,/u/) et avec différents niveaux d'effort articulatoire. Les signaux EMG de surface de cinq muscles péri-oraux ont été acquis simultanément avec une vidéo des lèvres. Les mouvements de parole ont été segmentés en quatre phases à l'aide du signal cinématique de distance interlabiale. Des patrons d'activation musculaire semblables ont été observés chez les quatre locuteurs, quels que soient la consonne et le contexte vocalique. Enfin, les activités du muscle dépresseur de la lèvre inférieure et du mentalis dans certaines phases du mouvement semblent être de bons descripteurs de l'effort articulatoire.

\section{ABSTRACT}

Our goal is to show the interest of distinguishing several phases in the gesture underlying the production of labial consonants, including during the occlusion, where lips are essentially static, in order to better understand their control. Four adult speakers were recorded, while producing logatoms including the consonants $/ \mathrm{p} /$ and $/ \mathrm{b} /$, followed by 3 vowels $(/ \mathrm{a} /, / \mathrm{i} /$ or $/ \mathrm{u} /)$ and with increasing levels of articulatory effort. Five surface EMG signals targeting five orofacial muscles involved in several lip movements were recorded simultaneously with a video of the lips. The speech gestures were segmented into four phases, based on the interlabial distance. Similar muscle activation patterns were observed for the four speakers, regardless of the consonant $(/ \mathrm{p} / \mathrm{or} / \mathrm{b} /)$ and the vowel context. Finally, the activities of the lower lip depressor and the mentalis muscles in some movement phases appear to be reliable descriptors of articulatory effort.

MOTS-CLES : plosives, électromyographie, effort articulatoire, patrons d'activation musculaire KEYWORDS: labial stop consonant, electromyography, articulatory effort, muscle activation patterns

\section{Introduction}

La production des gestes de parole requiert une coordination complexe des gestes respiratoires, laryngés et articulatoires. Le déplacement des articulateurs (langue, lèvres...), en particulier, est contrôlé à partir du recrutement précis de plusieurs muscles orofaciaux. La connaissance détaillée 
des muscles impliqués dans ces gestes, et des efforts physiologiques associés, serait d'un grand intérêt pour la modélisation de la production et la compréhension de divers troubles articulatoires. Cependant, si diverses méthodes sont communément utilisées en phonétique expérimentale pour caractériser le déplacement cinématique des articulateurs, l'électromyographie de surface reste encore très rarement utilisée pour caractériser les activités musculaires orofaciales sous-jacentes à ces gestes articulatoires.

Une des principales raisons à cela est certainement la difficulté de positionnement des électrodes. L'anatomie du visage est en effet fine et complexe: la densité musculaire est élevée, avec plusieurs muscles se superposant en particulier sur le pourtour des lèvres, et l'anatomie présente une importante variabilité inter-individuelle. De ce fait, les signaux EMG enregistrés avec les techniques d'électromyographie de surface bipolaire peuvent varier considérablement en fonction du positionnement des électrodes (voir par exemple : Beck et al., 2008 ; Campanini et al., 2007 ; Hogrel et al., 1998; Jensen et al., 1993; Roy et al., 1986), et une électrode peut capter l'activité d'un muscle proche, même s'il n'est pas directement situé sous cette électrode (on parle alors de « diaphonie »). O'dwyer et al. (1981) ont été les premiers à proposer des recommandations précises pour le placement des électrodes EMG sur le visage. Ils ont pu localiser précisément l'emplacement de fibres musculaires sous le derme du visage en réalisant l'acquisition des signaux EMG avec une technique intra-musculaire très invasive, mais permettant de capter les potentiels d'action moteurs directement sur les fibres musculaires, et en demandant à leurs participants de réaliser un ensemble de mouvements orofaciaux simples, silencieux, impliquant des recrutements musculaires bien identifiés. Leurs travaux se sont concentrés sur 10 muscles orofaciaux, parmi lesquels l'orbicularis oris, le mentalis (ou mentonnier) et le dépresseur de la lèvre inférieure, principalement recrutés dans les gestes de parole. Plus récemment l'avènement de l'électromyographie à haute-densité (HDEMG), qui exploite des matrices d'électrodes EMG de surface, a permis à Lapatki et al. (2010) d'apporter des compléments précieux aux connaissances sur la localisation des muscles orofaciaux et leur recrutement dans divers mouvements. Malheureusement, la forte variabilité anatomique inter-individuelle ne permet pas d'établir des règles universelles pour le placement des électrodes sur le visage, et des ajustements sont nécessaires pour chaque sujet lors du recueil des données.

Une autre source de difficultés relative à l'étude des activités musculaires orofaciales est la complexité de ces gestes et le fait qu'ils se composent en réalité de plusieurs sous-mouvements. La question se pose, par conséquent, de l'échelle de temps sur laquelle analyser les signaux EMG, et de la globalité vs. localité des descripteurs à en extraire. Certains auteurs (McClean et Tasko, 2003. Blair et Smith, 1986) se sont penchés sur cette question et ont ainsi cherché à décrire les patrons temporels d'activation des muscles orofaciaux lors de la production de la parole ou de mimiques faciales. McClean et Tasko (2003) ont ainsi proposé de décomposer les signaux de déplacement de la mandibule et des lèvres en une séquence de mouvements, grâce d'abord à la détection des pics de vitesse tangentielle, puis par le repérage des débuts et fins de mouvements correspondant aux minima de la vitesse tangentielle situés immédiatement avant et immédiatement après chacun des pics de vitesse. Plutôt que de mesurer l'énergie du signal EMG sur l'intégralité du mouvement, ils ont alors proposé de mesurer l'intensité électromyographique à partir du pic dans l'enveloppe EMG précédant un pic de vitesse de la mandibule ou des lèvres.

Dans cette présente étude des activations musculaires associées à la production des plosives labiales, nous proposons une méthodologie de segmentation des signaux cinématiques plus fine encore, dans laquelle nous définissons des phases correspondant à des portions de mouvements. Nous appliquons cette technique d'analyse pour décrire les patrons d'activation musculaire orofaciale ( 5 muscles) lors de la production de plosives prononcées par 4 sujets dans plusieurs conditions expérimentales. Dans une première partie nous présenterons le corpus et la méthode d'acquisition puis de traitement des données. Puis nous illustrerons l'intérêt de la segmentation en 
phases que nous nous proposons, d'abord par une analyse globale, puis en étudiant l'effet du contexte vocalique et de l'effort articulatoire sur les activations musculaires. Nous discuterons finalement ces résultats et conclurons.

\section{Matériel et Méthodes}

\subsection{Sujets et corpus}

Quatre sujets adultes ( 2 femmes, âge entre 21 et 33 ans) ont participé à l'expérience. Il leur était demandé de produire six logatomes : /ləpa/, /ləba/, /ləpi/, /ləbi/, /ləpu/, /ləbu/. Chaque logatome a été répété de manière isolée par séries de 5 items d'effort articulatoire (auto-évalué) croissant, à un rythme confortable et en insérant une courte pause entre chaque item. Les enregistrements duraient 30 secondes, permettant la production de cinq à six séries de logatomes à chaque fois.

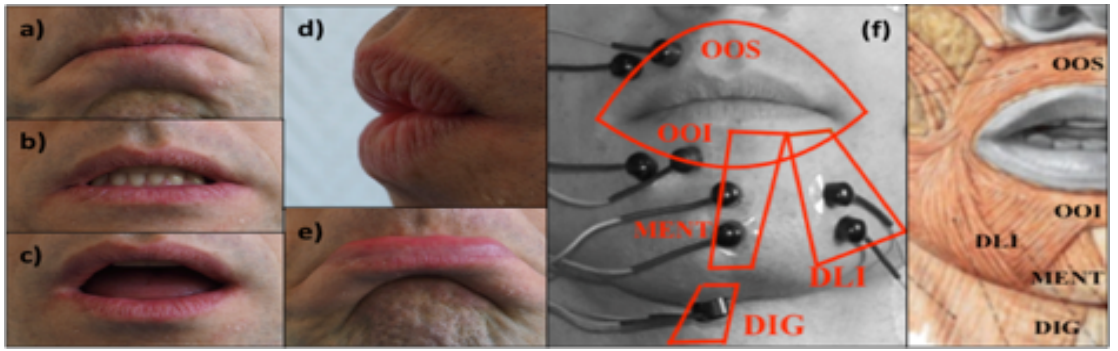

FIGURE 1 : Gestes orofaciaux silencieux utilisés pour valider le positionnement des électrodes EMG (a. compression labiale ; b.abaissement de la lèvre inférieure ; $\mathrm{c}$. abaissement mandibulaire ; d. protrusion labiale ; e. relèvement de la lèvre inférieure), localisation des 5 paires d'électrodes (f) et vue anatomique dans la région des lèvres.

\subsection{Matériel d'acquisition}

Cinq signaux EMGs de surface $(\mathrm{fe}=20 \mathrm{kHz}$ ) ont été simultanément acquis ciblant cinq muscles du pourtour des lèvres supposés intervenir dans le geste de production des consonnes occlusives labiales (Digastrique - DIG, Mentalis - MENT, Dépresseur de la lèvre inférieure - DLI, Orbicularis oris superior et inferior - OOS et OOI, cf Figure 1.f). Les conséquences du recrutement de ces muscles ont été abondamment décrites dans la littérature (voir par exemple O'dwyers et al., 1981). Le Mentalis (MENT) est recruté pour le relèvement de la lèvre inférieure tandis que le Dépresseur (DLI) est recruté pour l'abaissement de la lèvre inférieure. Ces deux muscles agissent de manières antagonistes lors du déplacement de la lèvre inférieure. Les deux parties, supérieure et inférieure, du muscle Orbicularis Oris (OOS et OOI) sont recrutées dans les mouvements de protrusion et de compression labiale. Le Digastrique (DIG), lui, est recruté dans l'abaissement de la mandibule. Les lèvres des sujets, maquillées en bleu pour faciliter la détection de leurs contours (Lallouache, 1991), ont été filmées de face à l'aide d'une caméra rapide (100 images/s). Le signal acoustique a été acquis à l'aide d'un microphone et d'un amplificateur de mesure Bruël \& Kjaer (Fréquence d'échantillonnage à $20 \mathrm{kHz}$ ) permettant de réaliser une mesure calibrée de l'intensité acoustique. Le signal audio et les signaux EMGs ont été acquis à l'aide du système BIOPAC MP160, qui par ailleurs assure la synchronisation de tous les signaux par l'envoi d'une impulsion de synchronisation à l'ensemble des dispositifs d'acquisition. 


\subsection{Segmentation des signaux cinématiques en 4 phases et extraction des descripteurs cinématiques.}

Dans la lignée des travaux de McClean \& Tasko (2003) nous avons procédé à une segmentation sur la base d'évènements mesurables sur les signaux cinématiques. Mais alors que McClean \& Tasko se sont essentiellement appuyés sur l'extraction des minima et maxima de la vitesse tangentielle, nous avons basé cette segmentation sur une analyse de la distance interlabiale et la détection des phases de contact entre les lèvres. En effet, lors de la production de consonnes occlusives labiales, il existe toute une phase pendant l'occlusion du conduit vocal où les lèvres sont en contact et où la vitesse de déplacement des lèvres est nulle ou très faible, alors même que l'activité musculaire est significative. Ce contact interrompt brutalement le mouvement, alors même que, de manière sousjacente, l'activation des muscles varie pour assurer l'occlusion et pour préparer le geste d'ouverture. C'est pourquoi nous avons observé la compression des lèvres durant la phase de contact et repéré des évènements temporels sur la base de la variation de la distance interlabiale et non du mouvement des lèvres. Nous avons préféré cette démarche à celle qui aurait consisté à segmenter directement les signaux EMG car elle fournit une information sur l'organisation temporelle des gestes phonétiquement pertinents, et nous semble donc bien caractériser les buts moteurs de la tâche pour lesquels les activations musculaires sont coordonnées.

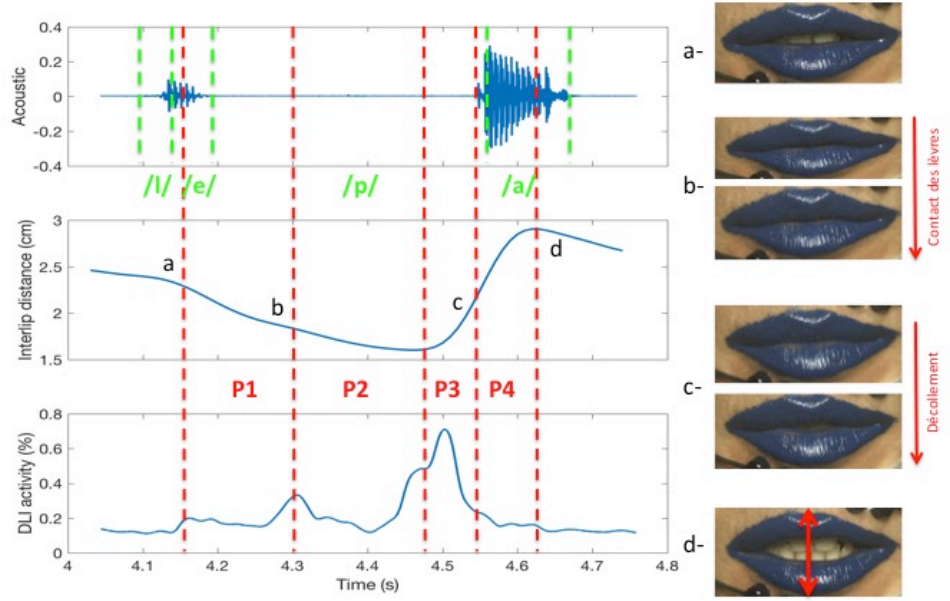

FIGURE 2 : Décomposition en 4 phases du mouvement labial lors de la production de la consonne $/ \mathrm{p} /$ dans /ləpa/. Les lignes pointillées vertes indiquent les limites de l'étiquetage phonétique de la séquence à partir du signal acoustique (cadre du haut); les lignes pointillées rouges marquent les évènements cruciaux pour la délimitation des phases, repérés sur le signal de distance interlabiale (cadre du mlieu): $\mathrm{a}=$ début de décroissance avant l'occlusion, $\mathrm{b}=$ début de contact, $\mathrm{c}=$ fin de contact, $\mathrm{d}$ fin de croissance après l'occlusion. Le cadre du bas représente l'activité EMG du Dépresseur de la Lèvre Inférieure (DLI).

Pour cela, dans chaque image vidéo, le contour externe des lèvres a été extrait à l'aide d'une technique d'apprentissage statistique de la couleur qui reprend les principes proposés par Lallouache (1991). Ces contours nous ont permis de mesurer la distance interlabiale qui sépare l'arc de cupidon du point le plus bas de la lèvre inférieure (Fig 2, flèche rouge sur l'image « $\mathrm{d}$ » et tracé du milieu). Nous avons ensuite repéré automatiquement l'instant de début de décroissance de la distance interlabiale précédant l'occlusion consonantique (Fig. 2, étiquette et image «a ») et l'instant de fin de croissance suivant le relâchement de la consonne (Fig. 2, étiquette « d »). Puis dans l'intervalle [a d] nous avons automatiquement détecté l'instant où la distance interlabiale atteint son minimum, et manuellement repéré les instants de début (Fig.2, étiquette et images «b») et de fin (Fig2. 
Etiquette et images «c ») de la phase de contact interlabial. Ces quatre étiquettes et le minimum de la distance interlabiale définissent 4 phases dans le mouvement des lèvres qui est à la base de la production de la consonne : une phase P1 de fermeture vers l'occlusion (intervalle [a b]), puis, pendant l'occlusion, une phase $\mathrm{P} 2$ de compression (de l'étiquette « $\mathrm{b} »$ au minimum de la distance interlabiale) et une phase P3 de relâchement de la compression (du minimum de la distance interlabiale à l'étiquette « $\mathrm{c} »)$, et enfin, après l'occlusion, une phase $\mathrm{P} 4$ d'ouverture (intervalle [c d]).

Trois autres paramètres ont été mesurés sur la variation de la distance interlabiale : la valeur minimum de la distance, appelée « degré de compression » et les amplitudes des pics de vitesse situés de part et d'autre de la phase d'occlusion (vitesses de fermeture et de réouverture).

\subsection{Post-traitement des signaux EMG et mesure de l'activité musculaire par phase du mouvement}

Le spectre fréquentiel des signaux EMGs est basse fréquence (maximum $500 \mathrm{~Hz}$ ). C'est pourquoi ces signaux, acquis à la fréquence d'échantillonnage de $20 \mathrm{kHz}$, ont été sous-échantillonnés à $2 \mathrm{kHz}$. Ils ont ensuite été filtrés à l'aide d'un filtre passe-haut ( $\mathrm{fc}=20 \mathrm{~Hz}$ ), afin de réduire les composantes basse-fréquences dues aux artefacts de mouvements des électrodes sur la peau. Leur enveloppe redressée a ensuite été calculée sur 500 points.

Pour chaque enregistrement (comprenant donc 20-25 répétitions d'un même logatome avec des variations d'effort), l'amplitude moyenne du bruit de mesure a été estimée sur chaque signal EMG à partir des premières centaines de millisecondes où le sujet était au repos, puis elle a été soustraite de l'enveloppe redressée du signal. Dans un second temps, pour chacun des cinq muscles ciblés, nous avons calculé la distribution des amplitudes de l'enveloppe redressée du signal EMG sur l'ensemble du corpus, repéré le seuil en dessous duquel les activités enregistrées pouvaient s'apparenter à un résidu de bruit, et soustrait cette valeur à l'enveloppe redressée du signal EMG. Les enveloppes redressées et seuillées ont ensuite servi de base à l'estimation des activités EMGs (Fig2, cadre du bas) : pour chaque muscle, l'amplitude de ces enveloppes a été normalisée par rapport à l'amplitude maximale observée pour ce muscle sur l'ensemble du corpus, permettant ainsi de comparer par la suite les activités des différents muscles en les exprimant en \% des activités maximales observables sur chaque muscle. L'activité EMG sur une fenêtre temporelle d'intérêt a été définie comme l'intégrale de l'enveloppe redressée du signal EMG, divisée par la durée de la fenêtre. Nous avons ainsi mesuré l'activité EMG de chacun des cinq muscles lors des quatre phases (P1-P4) de chaque mouvement de production des occlusives /p/ ou /b/.

\subsection{Analyses statistiques}

Pour chaque muscle et chaque mouvement effectué, nous avons créé une variable réponse prenant comme valeur la phase dans laquelle l'activité musculaire est maximale. Nous considérons cette variable réponse comme une variable catégorielle ordonnée (4 niveaux : P1 à P4).

Nous avons testé l'influence du contexte vocalique (3 niveaux : /a/, /i/, /u/), du niveau d'effort (5 niveaux), du muscle (5 niveaux, DIG, DLI, MENT, OOI et OOS) et de leurs interactions sur cette variable réponse, à l'aide d'une régression ordinale avec effets aléatoires (package ordinal de $\mathrm{R}$ ). Nous avons utilisé une procédure de sélection pas à pas, utilisant des tests de rapport de vraisemblance, pour sélectionner les termes du modèle qui apportent de l'information significative. Nous avons ensuite effectué des comparaisons multiples (package mulcomp de R) pour tester, pour chaque muscle, la significativité des différences observée entre les contextes vocaliques. 
Enfin, des analyses de corrélation de Spearman ont été conduites pour examiner la corrélation entre l'activité de chacun des 5 muscles, dans chaque phase du mouvement, et les 3 descripteurs cinématiques du mouvement des lèvres (compression labiale, vitesses de fermeture et de réouverture).

\section{Résultats}

\subsection{Patrons d'activation musculaire orofaciale lors de la production de consonnes occlusives - Influence de la voyelle adjacente}

La figure 3 ci-dessus représente les activités musculaires moyennes (calculées sur toutes les consonnes labiales et tous les locuteurs) des 5 muscles d'intérêt, selon que la voyelle suivant la consonne est /a /, /i/, ou /u/. On note d'abord une importante similitude dans l'évolution de ces activités pour chacune des voyelles et pour l'ensemble des muscles à l'exception de l'OOS : l'activité EMG croit dès la phase $\mathrm{P} 1$ pour atteindre un pic très marqué dans la phase $\mathrm{P} 3$ avant de décroitre dans la phase P4. Le muscle OOS est lui essentiellement actif en phase P1 et P2, et devient très faiblement actif dès la phase $\mathrm{P} 3$, sauf quand la voyelle suivant la consonne est un $/ \mathrm{u} /$. Pour les voyelles non labiales /i/ et /a/ les muscles présentant les activités les plus fortes sont les muscles « ouvreurs » DIG, DLI et MENT. Lorsque la voyelle suivante est /u/, l'activité de ces trois muscles est sensiblement réduite et ce sont les muscles OOS et OOI qui semblent les plus activés.

Dans tous les cas on note la pertinence de la décomposition en 4 phases : les activités EMG de tous les muscles varient entre les phases $\mathrm{P} 2$ et $\mathrm{P} 3$, qui, rappelons-le, correspondent à l'occlusion labiale au cours de laquelle les lèvres, en contact, sont sensiblement immobiles (i.e. à vitesse quasi nulle).

Contexte /a/

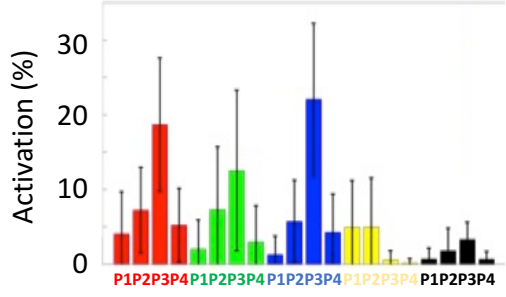

Contexte /i/

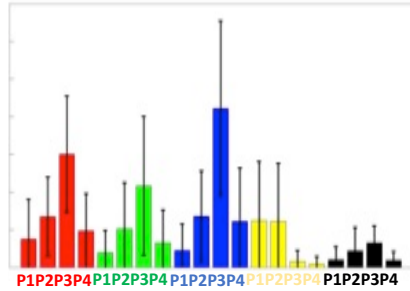

Contexte /u/

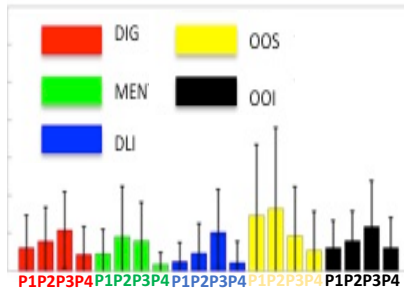

FigURE 3 : Evolution de l'activité EMG (exprimée en pourcentage de l'activité maximum dans tout le corpus) au cours des phases $\mathrm{P} 1$ à $\mathrm{P} 4$, pour les cinq muscles étudiés, moyennée sur l'ensemble de consonnes labiales et sur les 4 sujets, lorsque la voyelle suivant la consonne est /a/, /i/ ou /u/.

L'analyse statistique de ces données selon le modèle décrit en section 2.5 permet de préciser ce premier constat global. La phase du mouvement dans laquelle une activité musculaire maximale est observée dépend significativement du muscle considéré, du contexte vocalique, du niveau d'effort, ainsi que de l'interaction entre le muscle considéré et le contexte vocalique ( chisq $(8)=188.46, p<0.0001)$, et de l'interaction entre le muscle considéré et le niveau d'effort (chisq(16)=94.213, p<0.0001). En contexte /a/ et /i/ les amplitudes des activités musculaires sont du même ordre de grandeur, tandis que l'amplitude des activités musculaires est plus faible pour le contexte vocalique $/ \mathrm{u} /$ ). 


\subsection{Corrélations entre l'activité musculaire et le déplacement des lèvres}

Les analyses de corrélation entre l'activité de chacun des 5 muscles dans les 4 phases du mouvement, et les 3 descripteurs cinématiques du déplacement des lèvres (compression labiale, vitesse de fermeture, vitesse de réouverture) sont synthétisées sur la Figure 4. Celle-ci représente, pour chaque muscle et chaque phase du mouvement, la distribution des 24 coefficients de corrélation (Rho de Spearman) observés pour les 6 logatomes et les 4 locuteurs (chaque corrélation étant calculée sur environ 40 occurrences d'un même logatome). Cette représentation permet de repérer les cas pour lesquels une forte corrélation positive $(>50 \%)$ est très fréquemment ou quasi-systématiquement observée entre l'activité d'un muscle dans une phase du mouvement, et l'un des 3 descripteurs cinématique du mouvement (distributions présentant un pic très marqué autour de $75 \%$ ), et les cas pour lesquels l'activité musculaire n'est que peu corrélée, ou avec un degré de corrélation très variable, avec les descripteurs du mouvement.

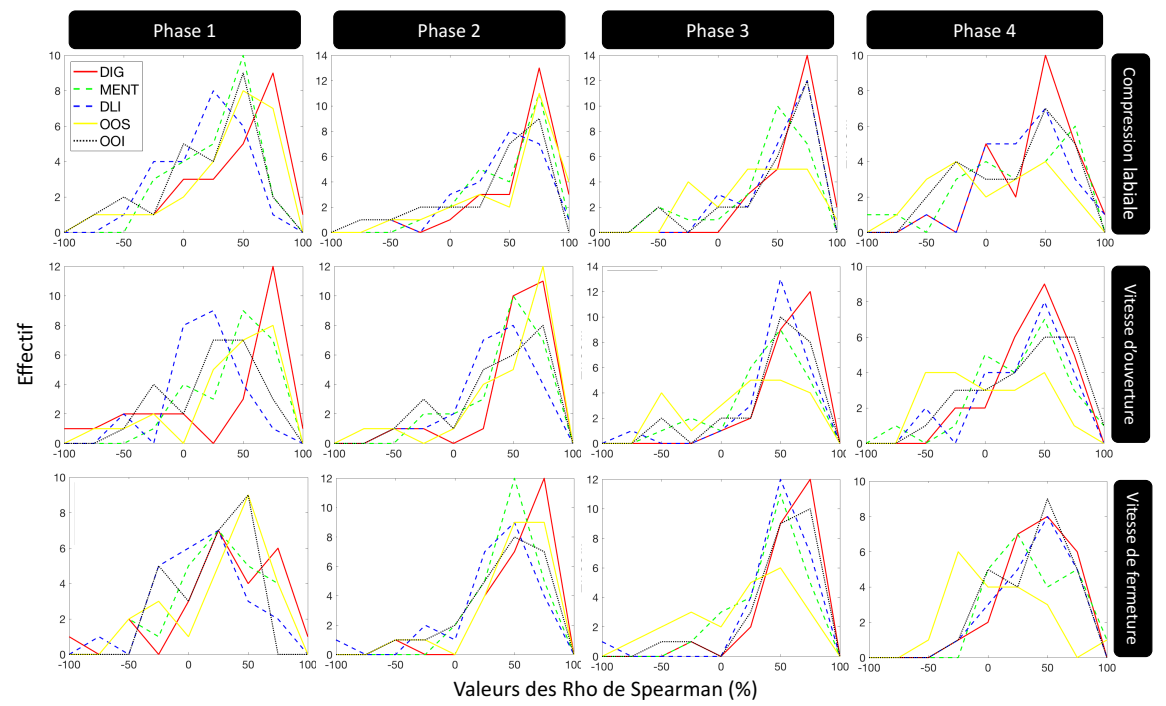

FIGURE 4 : Distribution des coefficients de corrélation (Rho de Spearman) entre l'activité de chacun des 5

muscles (DIG, MENT, DLI, OOS et OOI) dans les 4 phases du mouvement, et les 3 descripteurs cinématiques du déplacement des lèvres (compression labiale, vitesses de fermeture et de réouverture labiale).

Parmi les différentes corrélations d'intérêt, on note en particulier, que la compression labiale corrèle fortement, et de façon très reproductible, avec l'activité du MENT et de l'OOS en phase 2 du mouvement. On remarque également que la vitesse de ré-ouverture labiale corrèle fortement, et de façon très reproductible, avec l'activité du DIG en phase 3 du mouvement. Ces 3 activités musculaires repérées pourraient donc être de bons candidats comme descripteurs physiologiques de l'effort articulatoire. Ces pistes seront explorées plus en détails dans une prochaine base de données sur davantage de locuteurs.

\section{Discussion}

Pour les voyelles non labiales /i/ et /a/, l'analyse globale de la variation de l'activité des 5 muscles de la phase P1 à la phase P4 suggère que le muscle OOS (actif en phase P1 et P2) joue un rôle majeur dans la fermeture labiale, de l'initiation du mouvement jusqu'à la compression maximale, alors que le DIG, le DLI et le MENT, qui ont une activité croissante jusqu'en phase P3 
jouent un rôle majeur dans le geste de réouverture. Ces observations sont cohérentes pour les muscles OOS, DIG et DLI avec les connaissances générales sur le rôle fonctionnel de ces différents muscles. En revanche, elles sont surprenantes pour le MENT qui est plutôt considéré comme un muscle élévateur de la lèvre. Deux explications potentielles à cette contradiction peuvent être formulées. La première serait que les signaux EMG collectés sur l'électrode du MENT soient en fait contaminés par les potentiels d'action des muscles abaisseurs adjacents, en particulier le DLI (phénomène de diaphonie). La seconde serait qu'il existe un mécanisme de coactivation des muscles élévateurs et abaisseurs de la lèvre pour garantir une meilleure stabilité du geste d'ouverture, dont on sait qu'il est crucial pour la production des plosives. Cette hypothèse serait cohérente avec le concept de « synergie » entre muscles antagonistes proposé par Latash (2007), observé de manière quasi-systématique dans les mouvements du bras, et qui permettrait de stabiliser les mouvements.

Il est intéressant de noter que pour la voyelle /u/ les muscles les plus actifs sont le OOS et le OOI qui jouent un rôle majeur (cf par exemple Nazari et al., 2011) sur l'arrondissement des lèvres, crucial pour la voyelle /u/. L'activation plus forte de ces deux muscles débute bien avant le geste de la consonne vers la voyelle. On note donc au niveau musculaire l'effet d'anticipation du geste d'arrondissement vocalique dans la consonne, déjà largement observé sur le plan cinématique (Noiray et al., 2011). On note aussi, que pour cette voyelle, l'OOS est actif en phase P3, ce qui est cohérent avec le rôle que nous attribuons à son activation pour la production de l'arrondissement vocalique.

De manière générale, il est important de noter qu'aucun des muscles considérés dans cette étude n'est actif en phase P4, qui est la phase d'ouverture où se produisent le bruit de plosion et les transitions formantiques vers la voyelle, dont la littérature a montré qu'ils sont des facteurs cruciaux de l'intelligibilité consonantique. Cela suggère que les muscles responsables du mouvement des lèvres lors de la production de la consonne ne conduisent pas le mouvement d'ouverture, mais déterminent les caractéristiques dynamiques des lèvres pour que leur vitesse d'ouverture et leur rigidité permettent les bonnes interactions entre les tissus et l'air pour donner les phénomènes aérodynamiques nécessaires à la réalisation de la consonne.

\section{Conclusion}

Les données présentées dans cet article ont permis de :

1. décrire temporellement l'activation musculaire de chaque muscle péri-oral ;

2. montrer l'intérêt d'une analyse temporelle plus détaillée de l'activité musculaire lors de la production de parole, ici de consonnes occlusives ;

3. identifier des descripteurs physiologiques de l'effort articulatoire, reproductibles entre les locuteurs et les segments de parole produits. Ainsi, trois descripteurs parmi d'autres possibles s'avèrent pertinents: l'activité des muscles MENT et OOS au cours de la phase de compression labiale (P2), et l'activité du muscle DLI au cours de la phase de relâchement de la compression (P3).

\section{Remerciements}

A Thomas Hueber de Gipsa-lab pour ses conseils avisés lors de la réalisation du programme de détection des contours labiaux.

Cette recherche est financée par l'Agence Nationale de la Recherche (Projet StopNCo : Effort et coordination dans la production des consonnes occlusives ; ANR-14-CE30-0017; Maëva Garnier). 


\section{Références}

BeCK T.W, Housh T.J, CRAMER J.T, WeIR J.P (2008). The effects of electrode placement and innervation zone location on the electromyographic amplitude and mean power frequency versus isometric torque relationships for the vastus lateralis muscle. Journal of Electromyography and Kinesiology, 18, 317-328.

BLAIR C, SMITH A (1986). EMG recording in human lip muscles : can single muscles be isolated ? Journal of Speech and Hearing Research, 29, 256-266.

CAmpanini I, Merlo A, Degola P, Merletti R, Vezzozi G, Farina D. (2007). Effect of electrode location on EMG signal envelope in leg muscles during gait. Journal of Electromyography and Kinesiology, 17, 515-526.

HogRel J.-Y, DUCHÊNE J, MARINI J.-F (1998). Variability of some SEMG parameter estimates with electrode location. Journal of Electromyography and Kinesiology, 8, 305-315.

Jensen C, VAsseljen O, WestgaArd R.H, (1993). The influence of electrode position on bipolar surface electromyogram recordings of the upper trapezius muscle. European Journal of Applied Physiology, 67, 266-273.

Lallouache, M. T. (1991). Un poste «Visage-parole» couleur. Acquisition et traitement automatique des contours des lèvres. Thèse de Doctorat, Institut National Polytechnique de Grenoble, Grenoble, France.

LapatKi B.G, Oostenveld R, VAn Dijk J.P, Jonas I.E, Zwarts M.J, STEGeman D.F (2010). Optimal placement of bipolar surfaceEMGelectrodes in the face based on single motor unit analysis. Psychophysiology, 47, 299-314.

LATASH M.L, SCHOlZ J.P, SCHÖNER G. (2007). Toward a New Theory of Motor Synergies. Motor Control, 11, 276-308.

McClean M.D, TASKo S.M, (2003). Association of Orofacial Muscle Activity and Movement During Changes in Speech Rate and Intensity. Journal of Speech, Language, and Hearing Research, 46, 1387-1400.

NaZAri, M. A., Perrier, P., Chabanas, M., PAyan, Y. (2011). Shaping by stiffening: a modeling study for lips. Motor control, 15(1), 141-168.

Noiray, A., CATHIARD, M. A., MÉnard, L., Abry, C. (2011). Test of the movement expansion model: Anticipatory vowel lip protrusion and constriction in French and English speakers. The Journal of the Acoustical Society of America, 129(1), 340-349.

O’Dwyer N.J, Quinn P.T, Guitar B.E., Andrews G, Neilson P.D (1981). Procedures for verification . of electrode placement in EMG studies of orofacial and mandibular muscles. Journal of Speech and Hearing Research, 24, 273-288.

RoY S.H, De LuCA C.J, SCHNEIDER J, (1986). Effects of electrode location on myoelectric conduction velocity and median frequency estimates. The American Physiological Society, 67, $1510-1517$. 